\title{
Serial robots geometrical parameter identification using modified quantum behaved particle swarm optimization
}

\author{
Haomeng Tong \\ School of Information Engineering, Zhejiang University of Technology, Hangzhou 310023, China; \\ beny623@163.com
}

Keywords: positioning accuracy, quantum behaved particle swarm optimization, serial robot, geometrical parameter errors.

\begin{abstract}
Knowledge of stringent value of the robot end-effector position is necessary in several industrial applications such as macroscopic structure measurement and assemblage. However, industrial robots cannot meet this challenge because of numerous errors. This paper presents a new method using quantum behaved particle swarm optimization (QPSO) to calibrate the robot geometrical parameters to improve the positioning accuracy of serial robots. Firstly, the errors of the axes are analysed, then the QPSO is used to calculate the errors. Experimental results show that the proposed method can effectively identify the robot geometrical parameters.
\end{abstract}

\section{Introduction}

Robots are usually used to perform highly accurate tasks. But their low absolute accuracy makes them cannot meet the requirements. Therefore, the improvement of robot accuracy is important. There are five error sources to affect the robot accuracy, and the geometrical parameter errors of them are the dominant error source which accounts for 95 percent [1]. So the robot positioning accuracy can be improved by identifying the error correction parameters.

There are three kinds of methods to calibrate the geometrical parameters. Firstly, using the kinematics models such as the D-H [2], the MD-H [3], or the S-model [4] to calibrate. Secondly, using indirect methods to find the kinematics errors [5][6], the relationship between the robot and the camera is calculated and then get the kinematics errors. Thirdly, optimization methods such as genetic algorithms, artificial neural networks, particle swarm optimization algorithms [7-9] are used to get the kinematics errors. [10] proved that the particle swarm optimization algorithm had better optimization capability than other optimization algorithms.

This paper proposed the method of using QPSO to identify the geometric errors. Firstly, geometric parameter errors are analyzed. Secondly the QPSO algorithm is introduced, and the QPSO algorithm is used to identify the robot geometric parameter errors, and the results show that the QPSO algorithm can improve the robot absolute accuracy greatly.

\section{Error model of robot}

Kinematic model

This paper involves the IRB 1400, which is an serial articulated robot, and all of the joints are rotary joints. The robot dimensions and the link frame are shown as the Figure 2. The kinematic model of the robot is expressed by using DH parameters [2]. 


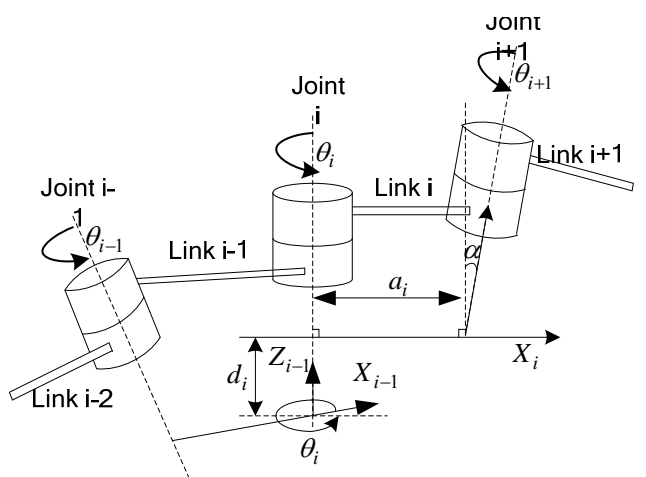

Fig. 1 Denavit-Hartenberg convention

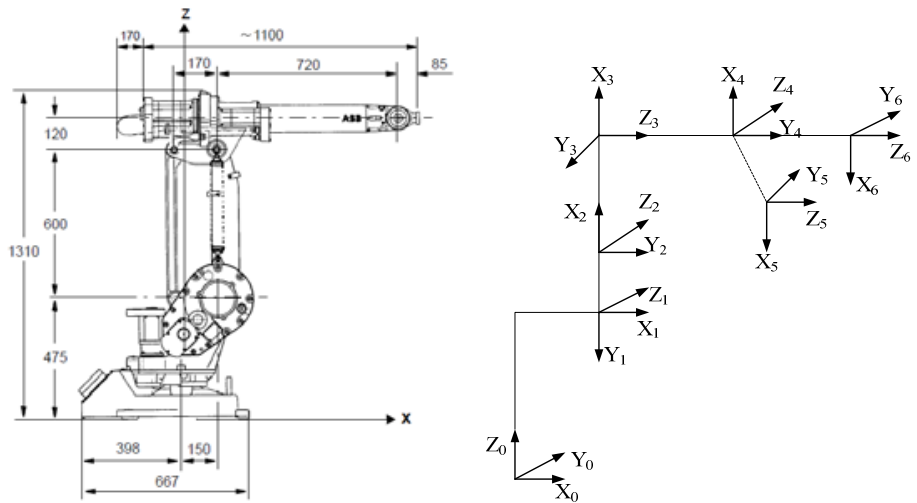

Fig. 2 Dimensions of IRB 1400 robot and link frame

The geometric parameters of the robot commonly do not match the designed goals. There are 24 parameters for the IRB 1400 robot, so there are 24 geometric parameter errors. But not all of them have the same contribute to the positioning errors. Therefore, differential motions of the end-effector related to each geometric parameter error will be analyzed first.

Suppose $\Delta \theta_{1}=0.01^{\circ}$, and other geometric parameter errors are 0. First, the first link is turned from $-170^{\circ}$ to $170^{\circ}$, and other links stay $0^{\circ}$. Such-and-such, all the six links move in the same way. The effect of the $\Delta \theta$ to the positioning errors are shown in figure 3 .

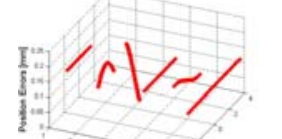

ins

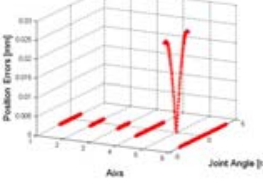

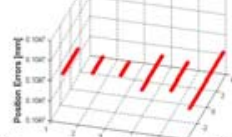

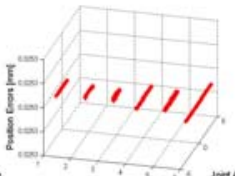

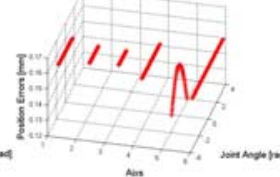

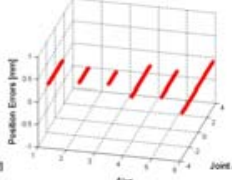

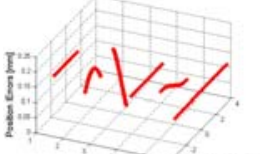

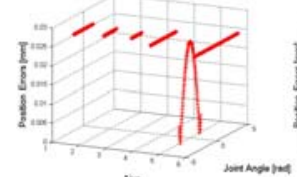

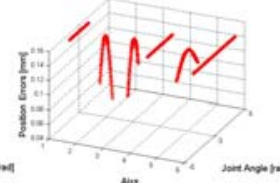
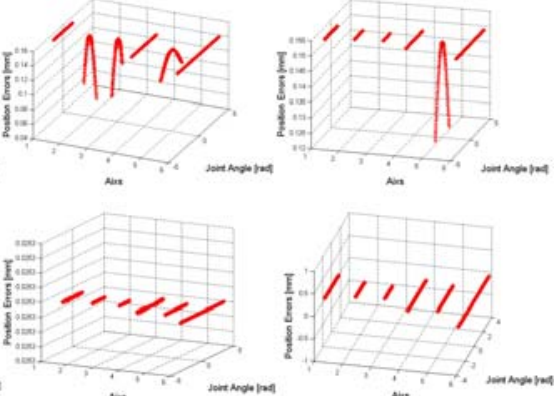

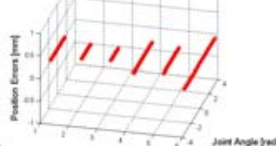

Figure 3 the effect of the $\Delta \theta$ to the positioning errors Figure 4 the effect of the $\Delta \alpha$ to the positioning errors
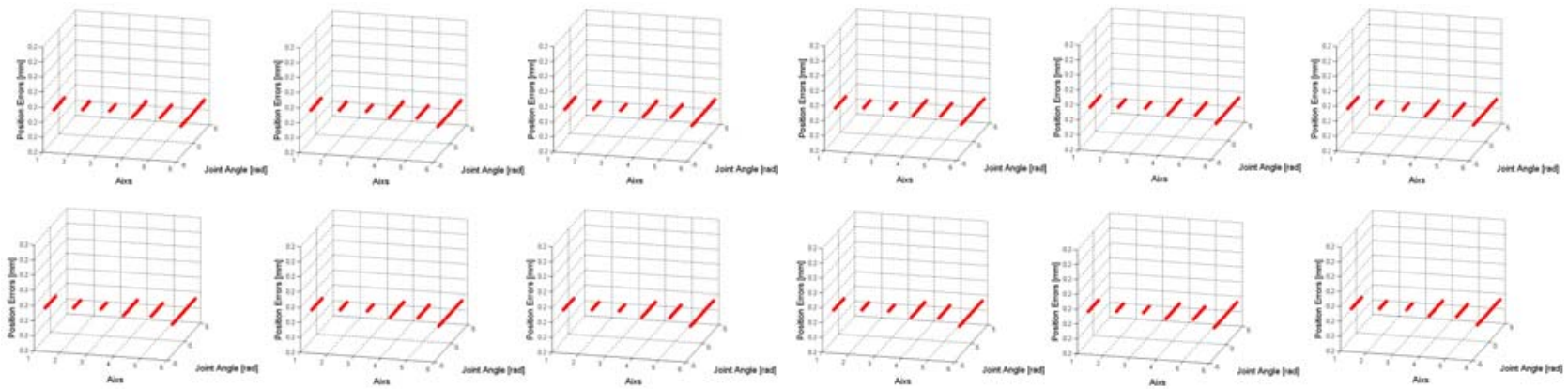

Figure 5 the effect of the $\Delta d$ to the positioning errors Figure 6 the effect of the $\Delta a$ to the positioning errors

In the same way, assuming the errors of each $\Delta \alpha$ is 0.01 , and the length parameter error $\Delta d$ and $\Delta a$ is $0.02 \mathrm{~mm}$, the positioning errors related to each parameter error are shown in figure 4 to 6.From figure 3 and figure 4 , we find that the joint parameter errors $\Delta \theta_{6}$ and $\Delta \alpha_{6}$ have no influence on the positioning accuracy, and $\Delta \theta_{5}, \Delta \alpha_{4}$ and $\Delta \alpha_{5}$ have very small influence which and be ignored. From figure 5 and 6 , we can see that the positioning errors caused by the length parameter errors are linear with the length parameter errors. Therefore, the discussion will be focused on 6 joint parameter errors $\Delta \theta_{1}, \Delta \theta_{2}, \Delta \theta_{3}, \Delta \alpha_{1}, \Delta \alpha_{2}, \Delta \alpha_{3}$ and 12 length parameter errors $\Delta d_{1} \ldots \Delta d_{6}$ and $\Delta a_{1} \ldots \Delta a_{6}$. 


\section{Quantum behaved Particle Swarm Optimization}

\section{Particle Swarm Optimization}

Because of the complexity of the robot kinematics system, it is difficult to use gradient and differentiable information to get the geometric parameter errors. But the search technique like particle swarm optimization can achieve the work. Particle Swarm Optimization (PSO)[11] has a powerful search capacity. But, the particles will be trapped into local optima after several iterations. To solve the problem, Quantum behaved PSO [12-13] optimization algorithm is proposed. In the QPSO algorithm, the particle has no fixed orbits and they can appear at any position at the solution space.

The particle position updating equations are as follows,

$$
\begin{gathered}
C_{j}=\left(C_{1}, C_{2}, \ldots C_{D}\right)=\frac{1}{M} \sum_{i=1}^{M} P_{i}=\left(\frac{1}{M} \sum_{i=1}^{M} P_{i, 1}, \frac{1}{M} \sum_{i=1}^{M} P_{i, 2}, \ldots \frac{1}{M} \sum_{i=1}^{M} P_{i, D}\right) \\
p_{i, j}=\varphi \cdot P_{i, j}+(1-\varphi) \cdot G_{i, j} x_{i, j}=p_{i, j} \pm \alpha \cdot\left|C_{j}-X_{i, j}\right| \cdot \ln [1 / u] \\
X_{i, j}=p_{i, j} \pm \alpha \cdot\left|C_{j}-x_{i, j}\right| \cdot \ln [1 / u], u \sim U(0 \sim 1)
\end{gathered}
$$

Where, $\varphi^{\sim} U\left(0^{\sim} 1\right), D$ is the dimension of the problem space; $M$ is the population size; $\alpha$ is the contraction expansion coefficient which is the only parameter in QPSO algorithm.

\section{Experiments}

The axis one joint angle is divided at intervals of 1 percent of the whole range. Axis one is moved to those angles while the rest axes stay zero. The corresponding angle data supplied by the robot controller are recorded. The same operation is carried out for the axis two to axis six. And 600 group geometric parameters are obtained. These parameters are nominal values and 18 parameter errors need to be identified as mentioned in section 2 . The true parameter values can be attained using the modified QPSO method.

When the robot axes are moved, the robot end-effector positions are measured at the same time. If the geometric parameters are accurate, the distance of any two positions moved by the robot should be the same with the distance measured by the laser tracker. So the objective function is,

$$
\left\|\left(X_{r}^{i}, Y_{r}^{i}, Z_{r}^{i}\right)-\left(X_{r}^{j}, Y_{r}^{j}, Z_{r}^{j}\right)\right\|=\left\|\left(X_{l}^{i}, Y_{l}^{i}, Z_{l}^{i}\right)-\left(X_{l}^{j}, Y_{l}^{j}, Z_{l}^{j}\right)\right\|
$$

The results applying nonlinear least squared (NLS) algorithm are displayed to compare with the QPSO algorithm, as shown in Table 1

Table 1 The results

\begin{tabular}{|l|c|c|c|}
\hline & Without optimization & NLS & QPSO \\
\hline Mean Error(mm) & 2.80 & 0.23 & 0.17 \\
\hline RMS Error(mm) & 12.09 & 0.18 & 0.12 \\
\hline Max Error(mm) & 18.96 & 1.29 & 0.44 \\
\hline
\end{tabular}

From table 1 we can see that, errors without any optimization are very large, and the results of the QPSO algorithm are the best.

\section{Summary}

A new method using QPSO algorithm is proposed to identify the robot geometric parameter errors. From the experimental results, the RMS error is less than $0.12 \mathrm{~mm}$ for the whole work space after the method is performed. Furthermore, this method provides a simple operative procedure, which makes it easy to be applied in industry. In addition, it has broad applications including numerous other kinds of serial robots. 


\section{References}

[1] Roth Z, Mooring B, Ravani B. An overview of robot calibration[J]. IEEE Journal on Robotics and Automation, 1987, 5(3): 377-385.

[2] Denavit J. A kinematic notation for lower-pair mechanisms based on matrices[J]. Trans. of the ASME. Journal of Applied Mechanics, 1955, 22: 215-221.

[3] Hayati S A. Robot arm geometric link parameter estimation[C]//Decision and Control, 1983. The 22nd IEEE Conference on. IEEE, 1983: 1477-1483.

[4] Stone H W. Kinematic modeling, identification, and control of robotic manipulators [M]. Springer Science \& Business Media (2012)

[5] Wang Y, Wei Z, Shao M, et al. A new method to calibrate robot visual measurement system[J]. Advances in Mechanical Engineering, 2013, 5: 548509.

[6] Wang Y, Ye X, Hao Y, et al. A New Calibration Method for Robot Visual Measurement System without Handeye Calibration Procedure[J]. 2015.

[7] Tang, Y. C and Xu, Y, Application of fuzzy Naive Bayes and a real-valued genetic algorithm in identification of fuzzy model. Information Sciences, 2005, 169, pp. 205-226.

[8] Wang XQ, Liu HD, Shi Y, et al. Research on Identification Method of Kinematics for Space Robot. Procedia Engineering 2012;29: 3381-3386.

[9] Asgharifard-Sharabiani P, Hannaneh A, and Nikkhah-Bahrami M. Calculation of helical gear's basic parameters using COP-data acquired by optical 3D digitizer. Proc. IMechE Part C: J. Mechanical Engineering Science 2011; 225:2953-2962.

[10] Hassan R, Cohanim BK, Weck OD, et al. A comparison of particle swarm optimization and the genetic algorithm. In: Proceedings of the 1st AIAA Multidisciplinary Design Optimization Specialist Conference, April, 2005.

[11] Kennedy $\mathrm{J}$ and Eberhart RC. Particle swarm optimization. In: Proceedings of the IEEE International Conference on Neural networks, Perth, Western Australia, 1995, pp. 1942-1948, (IEEE Press, Piscataway, NJ).

[12] Sun J, Feng B and Xu WB. Particle swarm optimization with particles having quantum behavior. In: Congress on Evolutionary Computation, 19-23 June 2004, pp.325-331.

[13] Sun J, Xu W, Liu J, Parameter selection of quantum-behaved particle swarm optimization. Advances in Natural Computation, 2005;3612: 543-552. 